\title{
Induced Monocytes-Derived HSCs (CD34+) with LPS Accelerated Homing Rat Bone Marrow-Mesenchymal Stem Cell (BM-MSCs, CD105) in Injured Pancreas
}

\author{
Fedik A. Rantam 1,2, Purwati1,3, Budi Setiawan4, Sony Wibisono3 ${ }^{3}$, Ferdiansyah1,5, \\ Joni Wahyuhadi1,6, Edward Mouli1,5, Dwikora N. Utomo' ${ }^{1,5}$, Heri Suroto ${ }^{1,5}$, Candra Bumi1 \\ ${ }^{1}$ Stem Cell Laboratory, Institute of Tropical Disease, Airlangga University, Surabaya, Indonesia \\ ${ }^{2}$ Virology and Immunology Laboratory, Department of Microbiology, Faculty of Veterinary Medicine, Airlangga \\ University, Surabaya, Indonesia \\ ${ }^{3}$ Tropic and Infection Division of Internal Medicine, School of Medicine, Airlangga University, Surabaya, Indonesia \\ ${ }^{4}$ Animal Surgery, Animal Hospital, Faculty of Veterinary Medicine, Airlangga University, Surabaya, Indonesia \\ ${ }^{5}$ Regenerative Medicine, Department of Orthopedic, Dr. Soetomo Teaching Hospital/School of Medicine, \\ Airlangga University, Surabaya, Indonesia \\ ${ }^{6}$ Department of Neurosurgery, Dr. Soetomo Teaching Hospital/School of Medicine, Airlangga University, \\ Surabaya, Indonesia \\ Email: fedik.ar@gmail.com
}

Received 13 February 2015; accepted 1 May 2015; published 11 May 2015

Copyright (C) 2015 by authors and Scientific Research Publishing Inc.

This work is licensed under the Creative Commons Attribution International License (CC BY).

http://creativecommons.org/licenses/by/4.0/

(c) (i) Open Access

\section{Abstract}

Investigating the function of combining induced rat monocytes-derived bone marrow-haemopoietic stem cell (rat BM-HSCs) with LPS and rat bone marrow-mesenchymal stem cell (rat BM-MSCs) was to analyze the acceleration of homing process mechanism in injured pancreas. Mononucleated stem cells were isolated from aspirated whole rat BM using ficoll and cultured in $\alpha$-MEM complete growth medium in $10 \mathrm{~cm}$ petridish. After two days, adherent cells after washing twice in petridish were added $\alpha$-MEM growth medium and then mesenchymal cells were characterized using CD105 marker in third passage and labeled PKH26. Then haemopoietic stem cells (HSCs) were isolated with magnetic beads CD34+ and differentiated in vitro, and then induced monocytes with LPS. Animal experiment used 28 male Wistar rats, and divided them into 4 groups. After transplantation combined, both cells between monocyte derived HSc (mHSCs) and rat BM-MSC were analyzed expression of pair box gen 4 (Pax4), pancreatic and duodenal homeobox (Pdx1), C-peptide using immunohistochemistry, then secretion of insulin and C-peptide analyzed using in-direct ELISA. Results showed that the expressions of Pax4, Pdx1, C-peptide found in the surface membrane cell

How to cite this paper: Rantam, F.A., Purwati, Setiawan, B., Wibisono, S., Ferdiansyah, Wahyuhadi, J., Mouli, E., Utomo, D.N., Suroto, H. and Bumi, C. (2015) Induced Monocytes-Derived HSCs (CD34+) with LPS Accelerated Homing Rat Bone Marrow-Mesenchymal Stem Cell (BM-MSCs, CD105) in Injured Pancreas. J. Biomedical Science and Engineering, 8, 333-344. http://dx.doi.org/10.4236/jbise.2015.85031 
of pancreatic cell, and secreted C-peptide and insulin were shown significant $(P<0.05)$ in transplanted group 2, 3 and 4, but in group 3 were transplanted with combined cells more dominant than non-combined cells. Conclusions suggested that combining of induced monocytes-derived HSCs and rat BM-MSCs has accelerated homing MSCs into injured pancreatic tissue.

\section{Keywords}

Induced Monocyte Derived HSCs, Rat BM-MSCs, Homing, Injured Pancreas

\section{Introduction}

The role of MSCs in the modulation of the immune response, immune system activity, and the body's response to inflammation and disease has been widely studied for many years, but homing mechanisms are still limited. MSCs both rely upon and co-create a network that facilitates constant communication between normal and damaged cells in the body [1]. MSCs might be metaphorically compared to a signaling system that has been extensively studied but remains not fully understood. In particular, the factors that trigger MSC responses and the tools required for MSCs to respond in a positive way to a particular insult to the body remain largely unknown. To effectively fight the fires in our bodies, an adequate supply of MSCs with high potential is needed to, metaphorically, act as firefighters. Properly culture-expanded and engineered MSCs with enhanced homing capability can ensure removal of the damaged cells and increase the rate of regeneration when the balance is disrupted in the body [2] [3]. A variety of strategies have been suggested to enhance the homing of MSCs based on their well-known characteristics [4]. On the other hand, the acute phase of injury, factors released from damaged tissues recruit blood cells and MSCs to repair the injured site. In this regard, substantial evidence indicates that infused MSCs have higher engraftment efficiencies within sites of inflammation or injury. Also it is demonstrated that inflammatory stimuli of allergic rhinitis induced the homing of intravenously administered hAdMSCs to cell-damaged areas. The evidences indicate that signals are required to recruit MSCs with high efficiency, which is critical for improving the clinical benefits of MSCs [5].

In this study, the acceleration of homing mechanism using induced monocytes-derived HSCs CD34+ in injured pancreas was analyzed, because activated monocytes can express many kinds of molecule signaling like CRCX4 and TNF- $\alpha$ that can induce migration of rat BM-MSCs to the injured area. Monocyte chemo-attractant (MCP)-1 is typically expressed at sites of inflammation and can thus represent a model homing chemokine, because MSCs have expressed the MCP-1 receptor and chemokine receptor (CCR) 2 on the cell surface membrane cell [6].

Multiple approaches are now being investigated to generate insulin-producing cells in vitro either by genetic engineering of $\beta$-cells or by utilizing various $\beta$-cell precursor cells and stem/progenitor cells with the ability to grow in vitro and to differentiate into insulin-producing cells and ultimately into $\beta$-cells [7]. The demonstration of plasticity of mesenchymal stem cell has been published and has led to their study in the treatment of diabetes [8]. In diabetics, $90 \%$ of insulin-secreting $\beta$-cells of the pancreatic islets of Langerhans are destroyed. Some investigators has demonstrated that insulin secreting cells ( $\beta$-cells or otherwise) could be transplanted into patients to help maintain blood glucose homeostasis, reduce the burden of diabetes-related complications, overcome the limitation of donor organs and provide benefit to millions of diabetics, and thus the replacement of insulin-secreting $\beta$-cells would be an ideal cure [8]. Studies of the growth, development and differentiation of bonemarrow mesenchymal stem cell (BM-MSCs) have been explored as a new clinically relevant cell type to repair injured tissue, and they can modulate immune response going to up-regulation and down-regulation like in the sepsis cases or ischemia. But the mechanism of accelerating homing modulation in injured tissue is still unclear. This research was to find the model of acceleration of homing mechanism that interacted between ligands and receptors of mesenchymal and monocytes-derived HSCs like chemo-attractant.

\section{Materials and Methods}

\subsection{Animal Experiment}

This experiment used male Wistar rats, weight 200 gram and age 3 mounths were obtained from Veterenary 
Farma, Surabaya, Indonesia who that undergoverment instituton has produced for vet vaccine, diagnostic and has breeding of some kinds animals laboratory. Four groups of 28 Wistar rats, first group for control and each of second to fourth groups were treated using aloxan injection in dose of $50 \mathrm{mg} / \mathrm{kg}$ intraperitoneally according to method of Purwati [8], and then fasted for $72 \mathrm{~h}$, and levels of C-peptide and insulin were measured by ELISA (Mercodia-Uppsala) to determine levels of $\beta$-islet pancreas damage. All animal experiments operation prosedure were conducted with institutional of ethics approval in accordance with Animal Care and Use Committee (ACUC) the Faculty of Veterinary Medicine, Airlangga University, Surabaya, Indonesia. Then research were design first group for control, second group were transplanted BM-MSCs, third group were transplanted mix cell of BM-MSCs and induced monocyte derived HSCs, fourth group were transplanted induced monocyte derived HSCs.

\subsection{MSCs Isolation and Culture of Rat Bone Marrow Stem Cell (BM-MSCs)}

Whole bone marrow were aspirated about $3 \mathrm{ml}$ then isolated using Ficoll 0.077 density (Invitrogen). After centrifugation by 1600 rpm, 30 min were isolated mononucleates cells from Buffy coat space and washed using growth medium serum free and followed centrifugation by $1600 \mathrm{rpm}$ in $10 \mathrm{~min}$. After that were cultured mononucleate cells in petridish $10 \mathrm{~cm}$ and growing in complete medium ( $\alpha$-MEM) (Invitrogen) according to modified method of Wolf [9]. In third passage of mesenchymal stem cells phenotype were characterized immunotyphing of using marker CD150 and flow cytometry according to modified method of Omori [10], and then labeled used PKH26 (Sigma, USA). Total of MSCs $2 \times 10^{5}$ were mixed with induced monocyte derived HSCs using ratio 1:5 for MSCs were used for transplanted to animal diabetic Mellitus.

\subsection{Immunocytochemistry}

This method was used to characterize of phenotype rat Bone-Marrow derived Mesenchymal Stem Cell (rBMMSC) specific CD105 and CD34+. Cells were fixed in ice-cold methanol at $-20^{\circ} \mathrm{C}$. Following two washes with PBS, the cells were blocked for 1 hour in PBS containing 1\% bovine serum albumin (Sigma-Aldrich Corp) and 0.1\% Triton X-100 (Sigma-Aldrich Corp) for 45 min and then incubated in the primary antibody (CD105, 1:100, Dako) for 2 hour at room temperature. After three washes with PBS, the cells were incubated in secondary antibody (fluorescein isothiocyanat-conjugated antibodies rabbit anti rat-Dako) for 1 hour, and after final wash, the cells were imaged using an Olympus inverted fluorescent microscope.

\subsection{Flowcytometry}

Flow cytometric analysis for phenotype including isotype control, was performed according to manufactures. Freshly isolated cells were incubated for 15 min with erythrocyte lysis buffer at $41^{\circ} \mathrm{C}$ then were cells washed with PBS, and incubated with Dil-ac-LDL (acLDL) $(10 \mathrm{mg} / \mathrm{ml})$ for 1 hour at $37^{\circ} \mathrm{C}$. Afterwards, cell were washed twice with plain medium, once with PBS, and fixed with $4 \%$ paraformaldehyde (PFA), and then cell were centrifuged at $1600 \mathrm{rpm}$ for 10 men and resuspended in PBS containing 0.2\% FCS and $2 \mathrm{mM}$ EDTA. For incubation with primary anti rat CD105-FITC cell were also fixed with 4\% PFA, resuspended in PBS containing $0.2 \%$ FCS and $2 \mathrm{mM}$ EDTA, and maintained at $41^{\circ} \mathrm{C}$ for $30 \mathrm{~min}$. After washing and incubation with secondary FITC-conjugate antibody was performed for 30 men at $41^{\circ} \mathrm{C}$ with sample. Labeled cells were analyzed by flowcytometry according to the guide of BD (BD Biosciences, San Jose, CA) and cellQuest pro analysis. The percentage of positively stained cells was determined after correction for the percentage of cell reacting with the secondary FITC antibody.

\subsection{Isolation and Culture of Monocyte-Derived BM-Haemopoietic Stem Cell (BM-HSCs) Inducing LPS}

Whole rat bone marrows were isolated using CD34+ magnetic beads (Invitrogen). After centrifugation by 1600 rpm in refrigerator centrifuge (Backman) was supernatant removed and resuspended in buffer elusion reagent (invitrogen) then repeated centrifugation by $1600 \mathrm{rpm}$ at $15 \mathrm{~min}$. After removing of supernatant were cells resuspended using complete medium $\alpha$-MEM and plated in petridish, and for immunotyphing using flow cytometry according to modified method of Tarja and Sharkis [11], Rantam [12]. Then differentiated monocyte were induced with purified-lipopolysacharide (LPS-Institute of Tropical Disease Universitas Airlangga). Three days of induced Cells were harvested and counted by haemocytometer for preparing and combining with rat BM- 
mesenchymal stem cell (BM-MSCs) through modified method of Belema-Bedada [6]. In these case were used ratio between rat BM-MSC and induced monocyte derived HSCs 1:5 for MSCs, then transplanted into DM-2 rat through intraperitoneally $2 \times 10^{5}$ of total cell according to modified method of Purwati [8].

\subsection{Immunohistochemistry Analysis of Pancreatic Repair}

Cell sections of pancreatic tissue were fixed with 4\% paraformaldehyde (Sigma) for 10 min. Immunohistochemistry was performed as previously described by Shuang-zhi [13] and Purwati [8]. The cell sections were incubated with rat monoclonal antibody anti-PDX-1 (1:200, Dako), anti-PAX-4 (1:200, Dako) and rat monoclonal antibody anti C-peptide (1:200, Dako). The positive cells were examined and mounted under inverted microscope.

\subsection{Indirect ELISA}

Microplates (96-well, Nunc) were coated with rat sera from each group overnight at $4{ }^{\circ} \mathrm{C}$, washed with PBS containing Tween 20 (PBST) and blocked for $1 \mathrm{~h}$ at room temperature with 1\% BSA. After three washes with PBST, the primary mAb anti C-peptide, insulin goat anti-rat were diluted appropriately in PBS 1:200 and incubated on the plates for $1 \mathrm{~h}$ at $37^{\circ} \mathrm{C}$. After three washes with PBST, horseradish peroxidase-conjugated anti-rat immunoglobulin was diluted in PBS 1:1500 and added to the wells. Following incubation for $45 \mathrm{~min}$ at $37^{\circ} \mathrm{C}$, the plates were washed three times with PBST, and ortho-phenylenediamine (OPD) substrate (Sigma) was added. The plates were incubated $10 \mathrm{~min}$ in the dark. After the addition of $1 \mathrm{M} \mathrm{HCl}$, the plate was read on an ELISA reader (BioRad) with the $450 \mathrm{~nm}$ wavelength. This method was modified from Tanaka [14] and Owen and Robert [15].

\subsection{Statistical Analysis}

Experimental data were presented as mean \pm SD. Statistical calculations were performed using SPSS13.0 (SPSS Inc., USA). For statistical analyses, were used the Student's t-test to compare data from normal donors with that obtained from injured pancreas and used a non-parametric test to compare the expression of pancreatic-specific markers. Statistical significance was defined as $\mathrm{p}<0.05$.

\section{Results and Discussion}

Rat bone marrow mesenchymal stem cells (rat BM-MSCs) were demonstrated previously by Purwati [8] for repair of $\beta$ pancreatic cell. To study the accelerated homing BM-MSCs were combined with induced monocyte derived HSCs, we first generated BM-MSCs and induced monocyte HSCs. Then analyzing of induced monocyte derived HSCs were demonstrated by expressing of TIR, MAC1, and CXCR4. Also the role of PDx1, Pax4 and C-peptide, insulin in injured pancreas.

\subsection{Phenotype Characterization of Mesenchymal Stem Cell CD105 and Haemopoietic Stem Cell CD34+}

Bone marrow mesenchymal stem cell (BM-MSCs) has many kinds of markers like CD73, CD90, CD105 [16], in this study were used CD105 marker and in third passage were identified using specific markers surface membrane cell anti CD150 labeled FITC. The results showed in Figure 1(d) about 89.1\% were positive expressed CD150, also expressed CD34+ haemopoietic stem cell about 93.1\% in Figure 2(c).

In these cases have been enough of MSCs count expressed CD105, these results indicate homogeneity and viability of BM-MSCs high. Although this marker was used mostly for proliferation and differentiation cells into osteoblast, chondroblast [12]. Expression of CD105 up to 80\% on the surface membrane cells have important role, that mean were these cells already to mix with induced monocyte derived HSCs for transplantation, because it had showed an character of multipotent mesenchymal stem cell.

The results showed in Figure 2(c), that count of mononucleate HSCs specific CD34+ about 93.1\% three days cultured cells. These results indicated that HSCs have enough homogeny and already to induce with LPS.

\subsection{Induced Rat Monocyte Derive Haemopoietic Stem Cell (HSCs)}

The data in Figure 3 showed that induced monocyte derive HSCs expressed TIR and CXCR4 on the surface membrane cells. These indicate that monocyte derive HSCs have been good function to attracted of MSCs as 


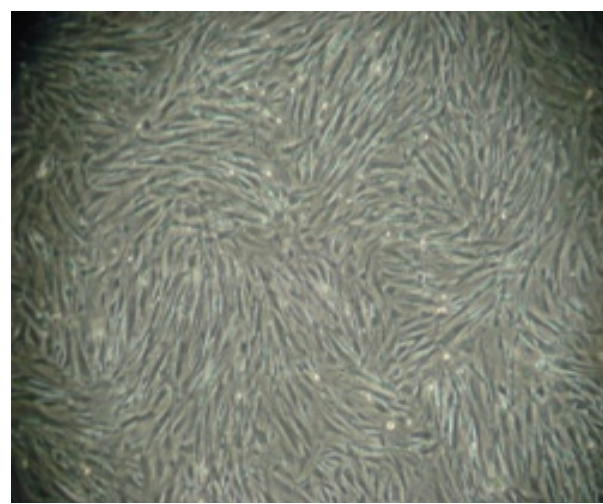

(a)

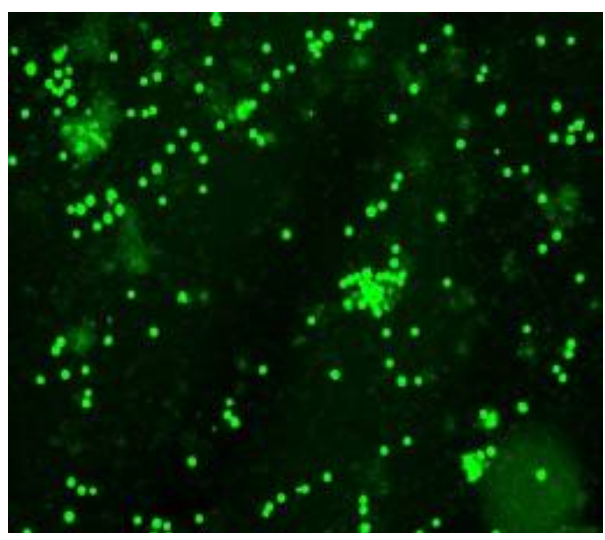

(c)

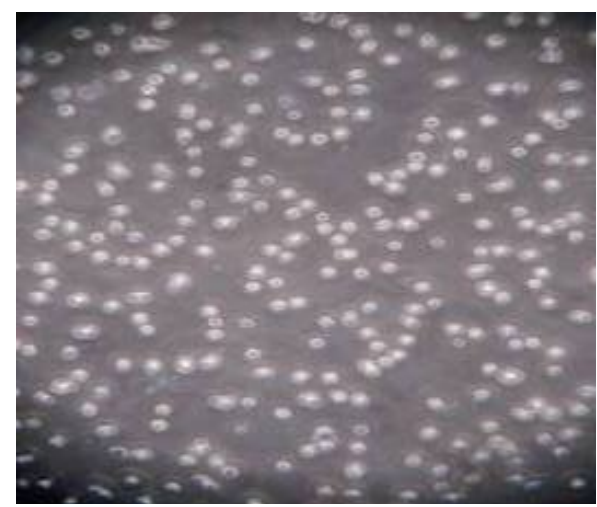

(b)

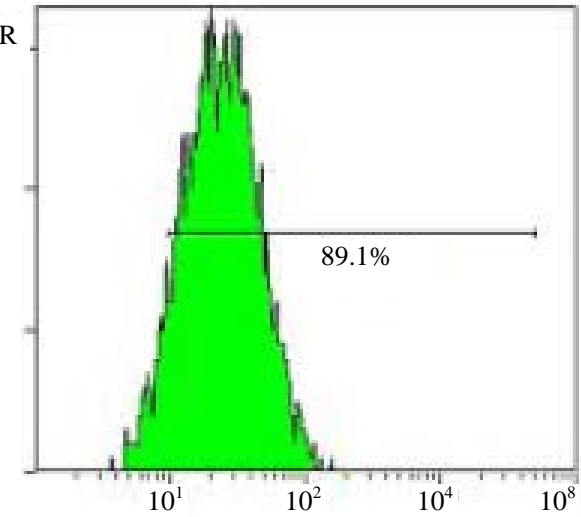

(d)

Figure 1. Analysis of bone marrow-mesenchymal stem cell (BM-MSCs) in third passage used CD105 marker surface membrane MSCs. (a) Monolayer of rat BM-MSCs; (b) Single cell of trypsinated monolayer MSCs; (c) Phenotype expression specific CD105 on the surface membrane MSCs; (d) Percentage of CD 105 expression were analyzed by flow cytometry. ×40 original magnifications.

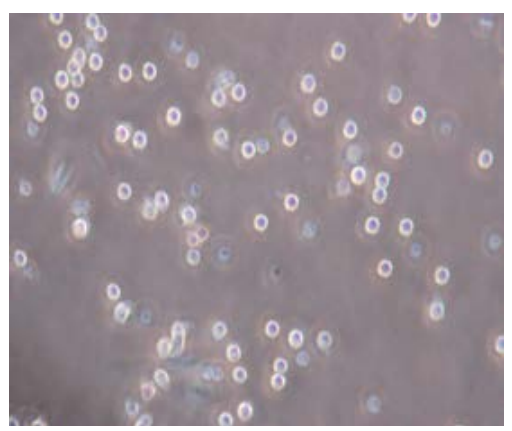

(a)

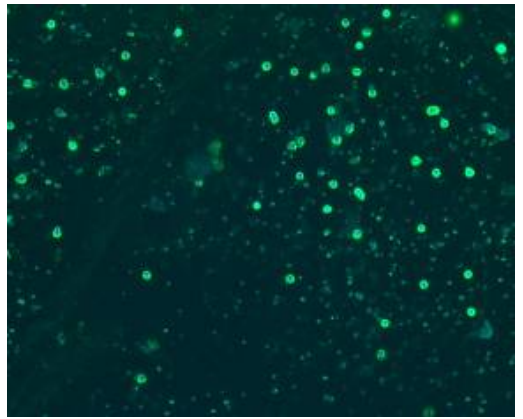

(b)

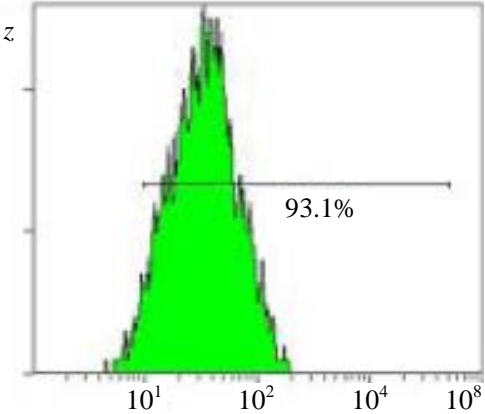

(c)

Figure 2. Rat bone marrow-haemopoietic stem cells (BM-HSCs). (a) Isolated HSCs of rat bone marrow using magnetic beads CD34+; (b) Three days of BM-HSCs cultured in complete medium $\alpha$-MEM were determinate of phenotyphing using specific marker CD34+ labeled FITC and analyzed by immunocytochemstry and; (c) Flow cytometry (FAC scabulary-BD). $\times 40$ original magnification.

once of migration factor during homing process. Although in generally showed that expression of CXCR4 relative highest $10 \%$ than TIR.

\subsection{Homing Process and PDX1-Pax4, and C-Peptide Expression}

Data in Figure 4 indicated that the healing process interacted with other molecule like chemokine, growth factor 


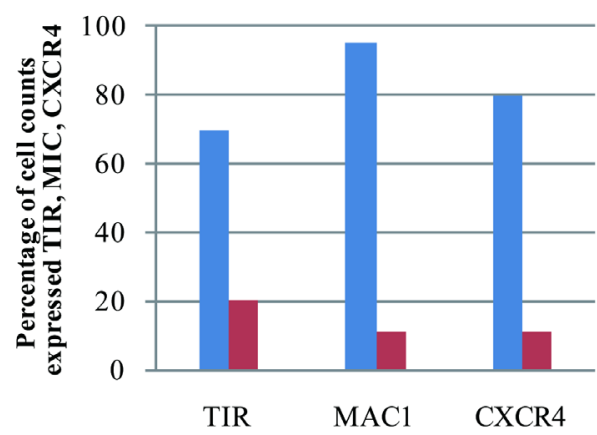

- After Induced LPS

- Before Induced LPS

(a)

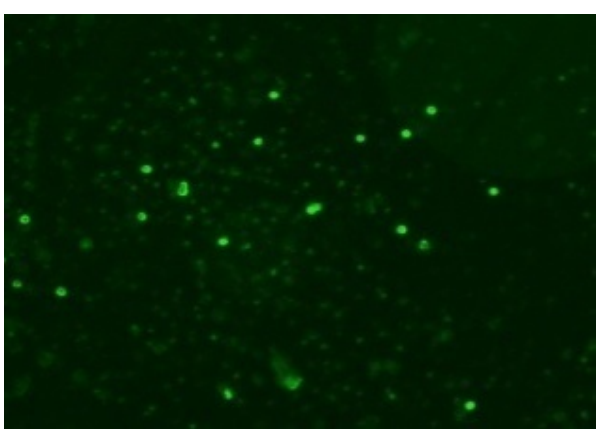

(b)

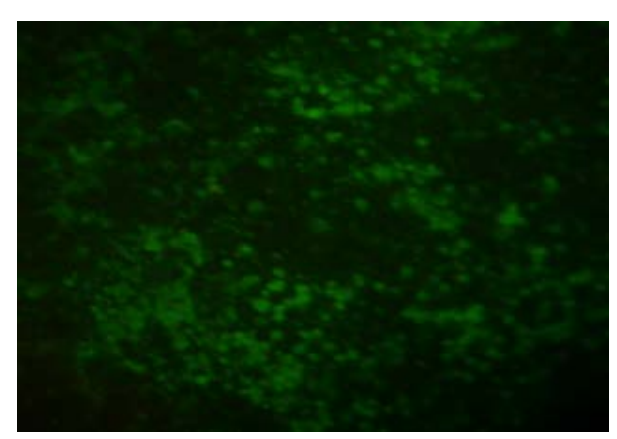

(c)

Figure 3. Analysis of protein expression in the surface membrane induced monocyte derived HSCs. (a) Diagram of expression toll interleukin receptor 1 (TIR-1), monocyte (MAC-1), chemokine receptor type-4 (CXCR4); (b) Differentiated monocyte derive HSCs were detected with MAC-1 labelled FITC; (c) Differentiated monocyte derives HSCs as negative control. $\times 40$ original magnifications.

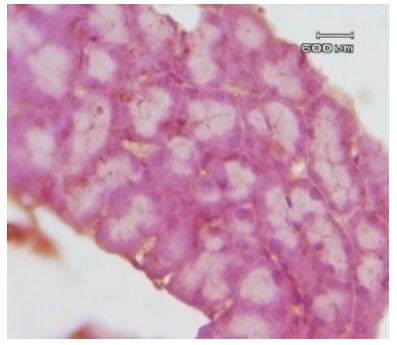

(a)

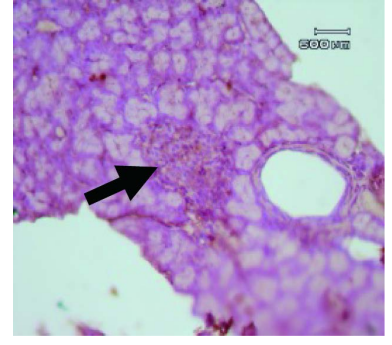

(b)

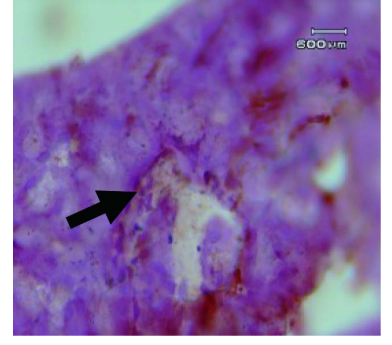

(c)

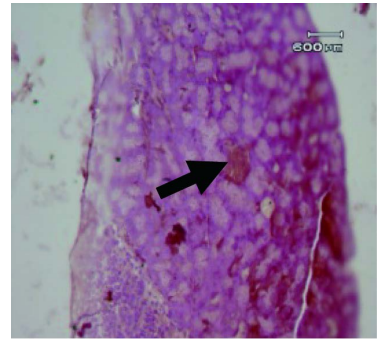

(d)

Figure 4. Immunohistochemistry analysis of homing and activated $\beta$-islet cells in pancreatic diabetic rat group 1 and group 3 , one week after transplanted mix cells of BM-MSCs and monocyte derive HSCs. (a) Normal rat pancreas were stained using rat anti-Pdx1; (b) Diabetic pancreatic rat were stained using rat anti-Pdx1, arrow showed Pdx1 expression; (c) Diabetic pancreatic rat were stained using rat anti-Pax4, arrow showed Pax4 expression; (d) Diabetic pancreatic rat were stained using rat anti C-peptide, arrow showed C-peptide expression. $\times 40$ original magnifications.

and extra cellular matric have most important role. Also expression of Pdx1, Pax4 and C-peptide have shown activated $\beta$ islet cells as transcription factor had good function in the homing as early in healing mechanism. The others hand, the expression of C-peptide in injured pancreatic like Figure 5. Have shown relative lower then compared to group 3 (Figure 4).

\subsection{Discussion}

Mesenchymal stem cell (MSC) is multipotent stem cell, that has a plasticity properties, and also have many kinds of protein as markers or signal expressed on the surface membrane cell have important role in communication between cell. CD105 is relative stable markers of MSC, because many kinds of markers can be used for detection of MSCs like CD90, CD73 and cells did not express CD34, CD11b, CD19, CD45 and HLA-DR [17]. 


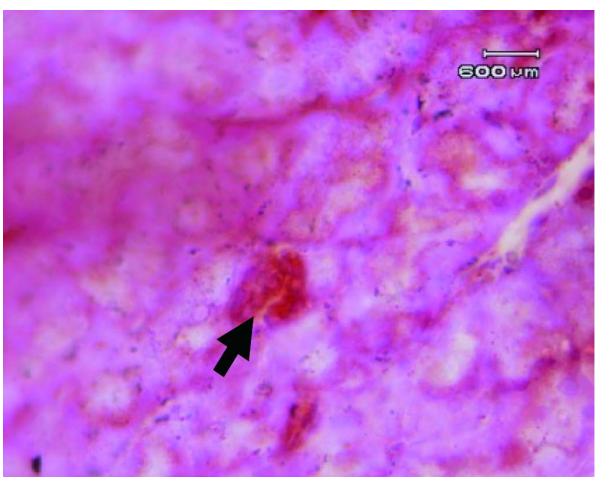

(a)

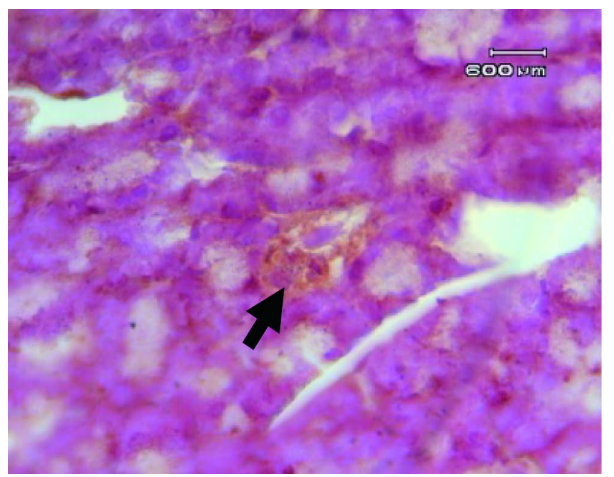

(b)

Figure 5. Immunohistochemistry analysis of homing and activated $\beta$-islet cells in pancreatic diabetic rat, one week after transplanted from animal experiment group and group 4. (a) Rat injured pancreatic group 2, one week after transplanted BM-MSCs only reacted with anti C-peptide, arrow showed C-peptide expression; (b) Rat injured pancreatic group 4, one week after transplanted induced monocyte derived HSCs only with anti C-peptide, arrow showed C-peptide expression. $\times 40$ original magnifications.

The other hand, we used CD34+ for isolation of haemopoietic stem cell from bone marrow. Because this marker was stable for mononucleat haemopoietic stem cell, and also were differentiated into monocyte, and characterized by monocyte (Mac1) markers. Induced monocyte derived HSCs (IM-HSCs) can express toll interleukine receptor (TIR) and CRCX4 like in Figure 3, this way it can be used as a chemotractant for accelerating of MSCs homing.

BM-MSCs have many kind of ligands expression and also IM-HSCs have many kinds of receptors with different function, but they were influenced high by microenvironment. This way the stem cell can derived into different type of desire cell. In this research, we used rat BM-MSCs with expression of CD105 positive 81.3\% in Figure 1(d) and HSCs expressed CD34 positive 91.3\% in Figure 2(c). The results showed that combining of MSCs and induced monocytes HSCs more better modulated to repair in injured pancreas than BM-MSCs and IM-HSCs only.

The primary goal of this study was to determine of the accelerated homing function by combining BM-MSCs and IM-HSCs, and characterized by expression of Pax4, Pdx1, and C-peptide on the surface membrane pancreatic cells.

Combining MSC and monocytes HSCs transplantation can increase activation of homing process than without combining stem cell. Other indicators showed TIR as signaling molecule, CXCR4 as adhesion molecule were increasing. The other meaning that both molecule especially monocytes HSCs can induce stem cells migration and adhesion into injured tissue. In order for MSCs to provide a pleiotropic immunomodulatory effect that is responsive to different stimulants such as cytokines and chemokines and targets different effectors cells such as T-cells, NK-cell and DCs, it seems reasonable for MSCs to employ both by direct and soluble mediators that coordinate for a multi-pronged approach to therapy [8].

Pax4 is positioned in the upper hierarchy among the $\beta$-cell transcription factors, and its expression in $\beta$-cell precursors results in the differentiation and maturation of $\beta$-cell. The results in Figure 4 showed expression of Pax4, that mean pax 4 as the first step to activated $\beta$-cell in injured pancreatic tissue. Because by inactivating of Pax4 in newborn Pax4 deficient mice, pancreases are almost entirely devoid of mature $\beta$-cell [18]. In vitro study have been reported that Pax4 over expression in mouse embryonic stem cells increases insulin-producing cells dramatically as compared to Pdx1 [19]. These suggesting Pax4 have important role in the healing process of injured pancreas.

Pdx1 expression like in Figure 4(b) showed that Pdx1 expressed co-populations of cells in injured pancreas, which possibility came from combined mix cell after transplantation to induce transdifferentiation into insulin producing cells. Pdx1 is transcription factor molecule can converts the hepatic-like cells into pancreatic precursor cells. Because it is this factor necessary for pancreatic development and $\beta$-cell maturation. The precursor cells can respond to glucose [18]. This way it was more important as markers to identify of precursor cells as transcription factor. Pdx1 appears to also play role in the fating of endocrine cells, encoding for insulin and so- 
matostatin, two pancreatic endocrine products, while repressing glucagon. Pdx1 pancreatic progenitor cell also co-express Nkx6-1, and these progenitor cell form the initial pancreatic buds, which further proliferate and branch in responses to FGF-10 signaling [20].

C-peptide is produced by a series of enzymatic cleavage of the precursor molecule preproinsulin and proinsulin. Preproinsulin is a precursor of proinsulin, is produced in the endoplasmic reticulum of pancreatic $\beta$-cell in respon to elevated blood glucose levels [20]. In this study, the results like in Figure 6 showed expression $\mathrm{C}$-peptide in population of pancreatic cells, but the secretion of C-peptide in serum significant $\mathrm{p}<0.05$. Table 1 suggested that glucose and insulin became increasing, and indicated that $\beta$-cell islet was survival, and were induced to switch in maturation of $\beta$-cell through paracrine effect of mesenchymal stem cell [21]. The capacity for self-renewal of post-natal murine $\beta$-cells have already study according to plasticity of MSCs [22], and also rat BM-MSCs has properties of the self renewal as multipotent stem cell. Through molecular and cellular pathways is highly likely. Endothelial signals have been linked with induction of pancreatic differentiation [23]. A dose-dependent effect has been noted [24], suggesting possible population expansion to be required prior to clinical transplantation. Finally, the demonstration of multi-step cell delivery being more efficient long-term [25] also underlines the possibility of introducing this principle in experimental and possibly clinical work.

In this research, pancreatic cells were injured with alloxan and then pancreatic cell was releasing stromal derived factor-1 (SDF-1). Alloxan would induce reactive oxygen species. The action of reactive oxygen species with a simultaneous massive increase in cytosolic calcium concentration causes rapid destruction of B cells [26].

Secretion of C-peptide, Insuline

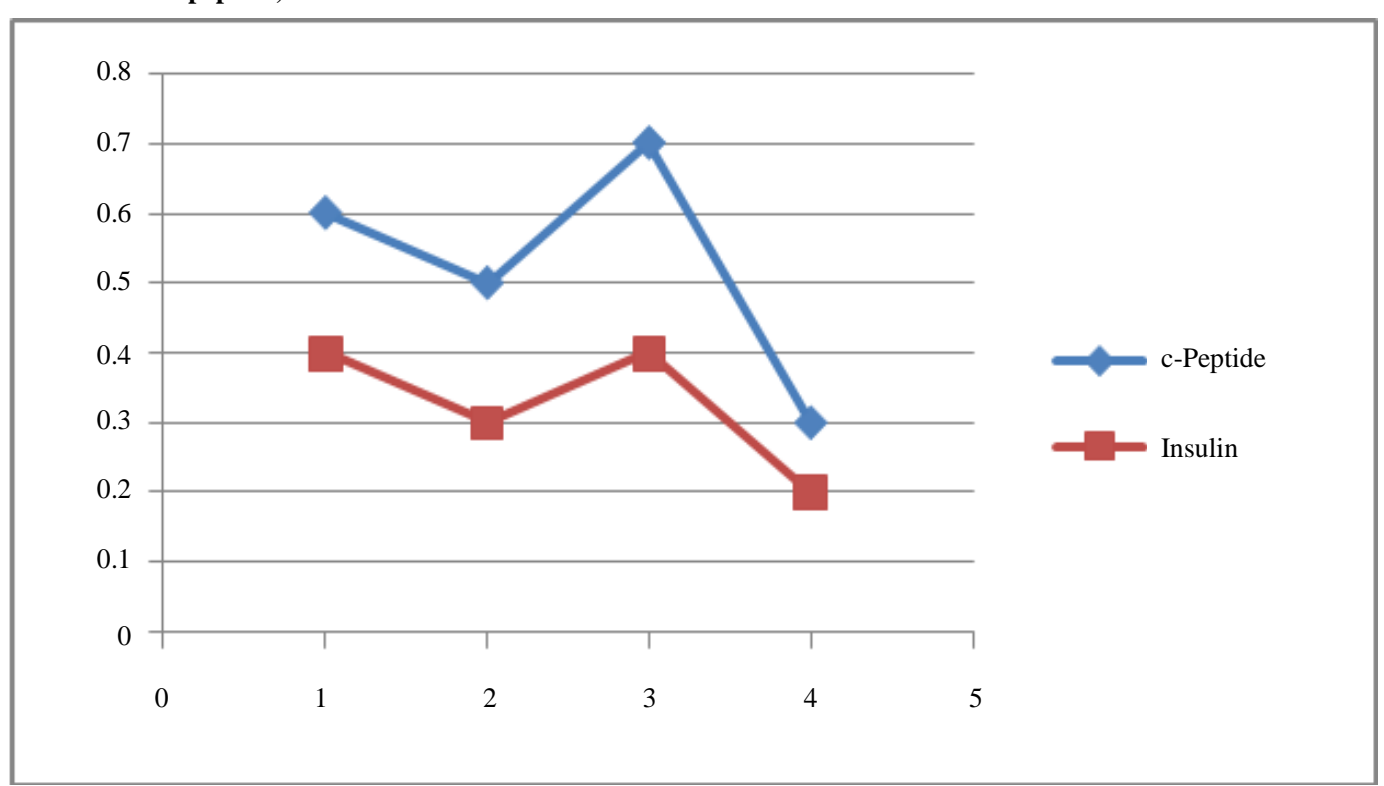

Figure 6. Secretion of C-peptide and insulin in serum, of all groups. Group 1: control healthy animal; Group 2: rat transplanted with BM-MSCs; Group 3: rat transplanted with mix cell of BM-MSCs and IM-HSCs; Group 4: rat transplanted with IM-HSCs. Group of negative control showed lower content of C-peptide and also insulin than group 3. Group 4 showed lowest than other group 2, 3, and group 2 showed relative in middle content than others results.

Table 1. Secretion of C-peptide and insulin content by rat injured pancreas, and one week after transplanted rat used BM-MSCs and induced monocyte HSCs (IM-HSCs).

\begin{tabular}{ccccc}
\hline Type & G1/plasebo & G2/BM-MSCs & G3/BM-MSCs and IM-HSCs & G4/Induced Monocyte HSCs \\
\hline C-peptide ng/ml & $1.02 \pm 0.05$ & $0.4 \pm 0.01$ & $1.00 \pm 0.05^{*}$ & $0.30 \pm 0.01$ \\
Insulin ng/ml & $0.49 \pm 0.05$ & $0.36 \pm 0.05$ & $0.42 \pm 0.02^{*}$ & $0.20 \pm 0.01$ \\
\hline
\end{tabular}

*Statistical significance from serum rat injured pancreas after transplanted combined between BM-MSCs and IM-HSCs. 
The chemokine SDF-1 are involved in cell migration, survival, and development of any cell types including hematopoeitic stem cells (HSC) and stromal stem cells (SSC) through CXCR4, during homeostasis CXCR4 highly expressed in HSC and low in SSC. In host defense and repair mechanism, SDF-1 and CXCR4 can recruitment of immature and maturing leukocytes from the bone marrow to damage organ like injured pancreatic cells. Homing stem cells in their niches regulate by the cross-talk between CXCR4 on hematopoietic cells and stromal cell-derived SDF-1 [27].

MSC have been shown to support hematopoiesis, it would be advantageous to co-transplant donor MSC with HSC to promote the rate of engraftment. Unfortunately, the transplant ability of marrow stromal elements remains controversial, with most studies showing that MSC, or stromal cells, have a limited capacity to reconstitute the marrow microenvironment. The fact that methods for isolation and amplification of MSC have been well studied and MSC home to BM with poor efficiency prompted us to find approaches to promote MSC homing and elucidate the mechanisms that guide homing of implanted MSC. Lately, CXCR4 was published to be expressed on the surface of MSC and BM stromal cells. The small proportion of MSC was expressed CXCR4, which contributed to their migration that capable of promoting migration to pancreatic islets in vitro. SDF-1 was reported could induce migration of human BM stromal cells in vitro and that CXCR4 might play a role in the engraftment of these cells in brain tissue of immunodeficient mice.

In this experiment we demonstrate for the first time that enforced surface expression of CXCR4, TIR and MAC-1 by inducing CD34+ with LPS was able to enhance in vivo homing on pancreatic islet. CXCR4 is known to be up regulated when human MSC are exposed to cytokines, including insulin-like growth factor- 1 , which is present in cell culture serum. Several studies now report that human MSC migrate in vitro in response to SDF-1, perhaps mediated by up regulation of intracellular CXCR4 molecules [28]. MSC homing in pancreatic cells was indicated by expression of Pdx-1, Pax4, and C-peptide that more highly expressed than control. Expression of Pdx-1 can trigger transdifferentiation of $\beta$ pancreatic cells to release insulin while expression of both Pax4 and C-peptide can induce both differentiation and maturation of $\beta$ pancreatic cells. Another experiment has been report that MSCs which do not express MAC-1, was also highly dependent on MAC-1 function [29]. MAC-1 expressing monocytes may induce MSC proliferation by an unknown mechanism. Outcome of CXCR4 signaling in some cells has been shown to be dependent on the concentration of SDF-1 [30]. Similar concentration-dependent mechanisms are emerging for effects of SDF-1 on activity of CD34 cells from different tissues. The chemokine SDF-1 plays a major role in migration, proliferation, differentiation, and survival of many cell types including human and murine hematopoietic stem/progenitor cells [31].

Beside MAC-1, LPS can induce hematopoietic stem cell expression of TIR/TLR4 through TRIF pathway [32]. MSC also express active TLR in various numbers depending on kind of source. TIR/TLR4 would trigger MSC for migration, proliferation and differentiation. Some groups have reported the effects of TLR activation on MSC differentiation with contradictory results [33]. Cell migration requires attachments to cells and matrix by adhesion molecules. Mac-1 is an adhesion molecule expressed by a variety of phagocytes including macrophages in inflammatory sites bacterial lipopolysaccharide (LPS)-induced migration of macrophages out of inflamed tissues and into the lymph nodes [34].

\section{Conclusion}

Homing stem cell can accelerate using combining between MSC and HSC. MSC has many kinds of molecular signaling or ligands on the cell surface, but monocytes HSC has many kinds of molecular ligands activated by cell and others cell to migration or interaction intracellular, and finally the cell can be easy to attachment to the other cell, then proliferation and differentiation and can integrate with host tissue. TIR and CCXR4 have an important role in homing process as attractants of MSCs. The accelerated mechanism of homing stem cell in injured pancreatic was showed in Figure 7. Thus, further research is required to understand the factors affecting the efficiency of MSCs migration and to determine strategies to remove harmful factors and improve homing of MSCs to the area of injury. New approach could mean smaller quantities of MSCs necessary for infusion, thereby attaining the intended therapeutic goal with greatest efficiency and efficacy. To achieve this goal, cell migration and tracking studies must be conducted in various in-vivo environments along with in-vitro laboratory studies. Through these studies, optimized culture conditions can be established to cultivate MSCs with enhanced homing ability and express the appropriate homing receptor. This is also essential to improve vascular conditions, so that introduced cells can easily migrate to damaged sites. 


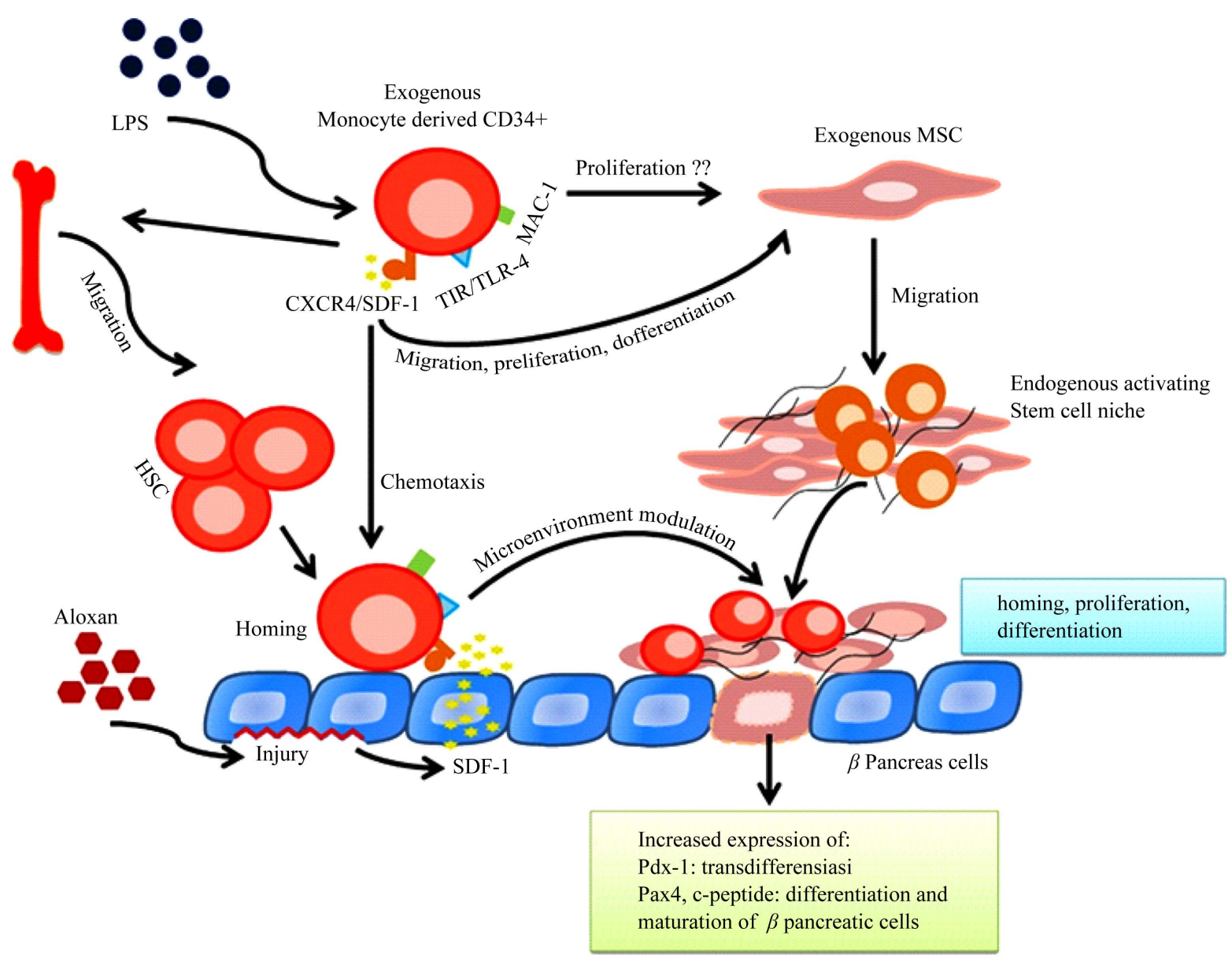

Figure 7. Mechanism of stem cell activity in injured pancreatic.

\section{Acknowledgements}

We thank to all researcher team at the Stem Cell Center, Institute of Tropical Disease Airlangga University Surabaya, East Java, Indonesia, Helen Susilowati, Eryk Hendrianto, Anas Prasetya Adi, Deya Karsari, Aristika Dinar Yanti, Nora Ertanti and Zahrotul Hasanah thank you for excellences of technical assistant.

\section{References}

[1] Rando, T.A. (2006) Stem Cells, Ageing and the Quest for Immortality. Nature, 441, 1080-1086. http://dx.doi.org/10.1038/nature04958

[2] Karp, J.M. and Leng Teo, G.S. (2009) Mesenchymal Stem Cell Homing: The Devil Is in the Details. Cell Stem Cell, 4, 206-216. http://dx.doi.org/10.1016/j.stem.2009.02.001

[3] Li, F., Huang, Q., Chen, J., et al. (2010) Apoptotic Cells Activate the "Phoenix Rising” Pathway to Promote Wound Healing and Tissue Regeneration. Science Signaling, 3, ra13. http://dx.doi.org/10.1016/j.stem.2009.02.001

[4] Rantam, F.A. (2003) Methods of Immunology. Airlangga University Press, Surabaya, 79-80.

[5] Kao, W.W., Liu, H. and Zhang, J. (2012) Umbilical Mesenchymal Stem Cells Can Differentiate to Assume Endothelial Cells Phenotypes. Association for Research in Vision and Ophthalmology (ARVO) Annual Meeting, Rockville.

[6] Belema-Bedada, F., Uchida, S., Martire, A., Kostin, S. and Braun, T. (2008) Efficient Homing of Multipotent Adult Mesenchymal Stem Cells Depends on FROUNT-Mediated Clustering of CCR2. Cell Stem Cell, 2, 566-575. http://dx.doi.org/10.1016/j.stem.2008.03.003

[7] Hess, D., Li, L., et al. (2003) Bone Marrow-Derived Stem Cells Initiate Pancreatic Regeneration. Nature Biotechnology, 21, 763-770. http://dx.doi.org/10.1038/nbt841

[8] Purwati, Rantam, F.A. and Wibisono, S. (2012) Autologous Mesenchymal Stem Cell for Treatment of DM2 in Rat. 
African Journal of Internal Medicine, 1, 1-8.

[9] Wolfe, M., Pochampally, R., Swaney, W. and Regar, R.L. (2008) Isolation and Culture of Bone Marrow-Derived Human Multipotent Stromal Cells (hMSCs). Methods in Molecular Biology, 449, 3-25. http://dx.doi.org/10.1007/978-1-60327-169-1_1

[10] Omori Y., Honmou O., et al. (2008) Optimization of a Therapeutic Protocol for Intravenous Injection of Human Mesenchymal Stem Cells after Cerebral Ischemia in Adult Rats. Brain Research, 1236, 30-38. http://dx.doi.org/10.1016/j.brainres.2008.07.116

[11] Tarja, A.J. and Saul, J.S. (2008) Isolation of Quiescent Murine Hematopoietic Stem Cells by Homing Properties. Mesenchymal Stem Cell, Humana Press, New York, 78-89.

[12] Rantam, F.A., Ferdiansyah, Nasronudin and Purwati (2009) Stem Cell Exploration. Airlangga University Press, Surabaya, 70-75.

[13] Huo, S.Z., Shi, P. and Pang, X.N. (2010) Culture and Identification of Human Amniotic Mesenchymal Stem Cells. Chinese Medical Sciences Journal, 25, 211-214.

[14] Tanaka, S., Endo, T., Aida, K., Shimura, H., Yokomori, N., Kaneshige, M., et al. (2004) Distinct Diagnostic Criteria of Fulminant Type 1 Diabetes Based on Serum C-Peptide Response and HbA1c Levels Onset. Diabetes Care, 27, 19361941. http://dx.doi.org/10.2337/diacare.27.8.1936

[15] Owen, W.E. and Roberts, W.L. (2004) Cross-Reactivity of Three Recombinant Insulin Analogs with Five Commercials Insulin Immunoassay. Clinical Chemistry, 50, 257-259. http://dx.doi.org/10.1373/clinchem.2003.026625

[16] Glettig, D. and Kaplan, D. (2013) Long-Term Phenotypic Characterization of Human Bone Marrow and Adipose Tissue Derived Mesenchymal Stromal Cells. Stem Cell Discovery, 3, 99-116. http://dx.doi.org/10.4236/scd.2013.32015

[17] Slater, K., Katie, P., Thomson, J., et al. (2013) Synthetic Peptide Coated Surface for Culture of Human Mesenchymal Stem Cell in a Defined and Xeno-Free Medium. Adult Stem Cell Therapy and Regenerative Medicine, Cleveland Marriott Downtown at Key Center, Cleveland, 19-21 August 2013, 53.

[18] Tang, D.Q., Cao, L.Z., Chou, W., Shun, L., Farag, C., Atkinson, M.A., et al. (2006) Role of Pax4 in Pdx1-VP16 Mediated Liver to Endocrine Pancreas Transdifferentiation. Laboratory Investigation, 36, 829-841. http://dx.doi.org/10.1038/labinvest.3700434

[19] Blysczcuk, P., Czyz, J., Kania, G., Wagner, M., Roll, U., St-Onge, L. and Wobus, A.M. (2003) Expression of Pax4 in Embryonic Stem Cells Promotes Differentiation of Nestin-Positive Progenitor and Insulin-Producing Cells. Proceedings of the National Academy of Sciences of the United States of America, 100, 998-1003. http://dx.doi.org/10.1073/pnas.0237371100

[20] Stanger, B.Z., Tanaka, A.J. and Melton, D.A. (2007) Organ Size Is Limited by the Number of Embryonic Progenitor Cells in the Pancreas but Not the Liver. Nature, 445, 886-891. http://dx.doi.org/10.1038/nature05537

[21] D’Amour, K.A., Bang, A.G., Eliazer, S., Kelly, O.G., Agulnick, A.D., Smart, N.G., et al. (2006) Production of Pancreatic Hormone-Expressing Endocrine Cells from Human Embryonic Stem Cells. Nature Biotechnology, 24, 13921401. http://dx.doi.org/10.1038/nbt1259

[22] Dor, Y., Brown, J., Martinez, O.I. and Melton, D.A. (2004) Adult Pancreatic Beta-Cells Are Formed by Self-Duplication Rather than Stem-Cell Differentiation. Nature, 429, 41-46. http://dx.doi.org/10.1038/nature02520

[23] Lammert, E., Cleaver, O. and Melton, D. (2001) Induction of Pancreatic Differentiation by Signals from Blood Vessels. Science, 294, 564-567. http://dx.doi.org/10.1126/science.1064344

[24] Ende, N., Chen, R. and Reddi, A.S. (2004) Effect of Human Umbilical Cord Blood Cells on Glycemia and Insulinitis in Type 1 Diabetic Mice. Biochemical and Biophysical Research Communications, 325, 665-669. http://dx.doi.org/10.1016/j.bbrc.2004.10.091

[25] Banerjee, M., Kumar, A. and Bhonde, R.R. (2005) Reversal of Experimental Diabetes by Multiple Bone Marrow Transplantation. Biochemical and Biophysical Research Communications, 328, 318-325. http://dx.doi.org/10.1016/j.bbrc.2004.12.176

[26] Szkudelski, T. (2001) The Mechanism of Alloxan and Streptozotocin Action in B Cells of the Rat Pancreas. Physiological Research, 50, 537-546. http://www.biomed.cas.cz/physiolres/pdf/50/50_537.pdf

[27] Dar, A., Kollet, O. and Lapidot, T. (2006) Mutual, Reciprocal SDF-1/CXCR4 Interactions between Hematopoietic and Bone Marrow Stromal Cells Regulate Human Stem Cell Migration and Development in NOD/SCID Chimeric Mice. Experimental Hematology, 34, 967-975. http://dx.doi.org/10.1016/j.exphem.2006.04.002

[28] Kyriakou, C., Rabin, N., Pizzey, A., Nathwani, A. and Yong, K. (2008) Factors that Influence Short-Term Homing of Human Bone Marrow-Derived Mesenchymal Stem Cells in a Xenogeneic Animal Model. Haematologica, 93, 14571465. http://dx.doi.org/10.3324/haematol.12553

[29] Osborne, J., Ledford, K., Parrish, C., Wong, A., Zeigler, F. and Bartel, R. (2011) Autologous Tissue Repair Cells from 
Bone Marrow Require Mac-1 Integrin Function for Expansion of both Hematopoietic Cells and Mesenchymal Stem Cells. Aastrom Biosciences, Inc., Ann Arbor.

http://vcel.com/wp-content/uploads/2012/09/Aastrom-Presents-Results-From-Two-Studies-of-Autologous-Bone-Marro w-Derived-ixmyelocel-T-sm.pdf

[30] Plett, P.A., Frankovitz, S.M., Wolber, F.M., Abonour, R. and Orschell-Traycoff, C.M. (2002) Treatment of Circulating CD34 ${ }^{+}$Cells with SDF- $1 \alpha$ or Anti-CXCR4 Antibody Enhances Migration and NOD/SCID Repopulating Potential. Experimental Hematology, 30, 1061-1069. http://dx.doi.org/10.1016/S0301-472X(02)00880-9

[31] Kollet, O., Petit, I., Kahn, J., Samira, S., Dar, A., Peled, A., et al. (2002) Human CD34 ${ }^{+}$CXCR4 ${ }^{-}$Sorted Cells Harbor Intracellular CXCR4, Which Can Be Functionally Expressed and Provide NOD/SCID Repopulation. Blood, 100, 27782786. http://dx.doi.org/10.1182/blood-2002-02-0564

[32] Takeda, K. and Akira, S. (2004) TLR Signaling Pathways. Seminars in Immunology, 16, 3-9. http://dx.doi.org/10.1016/j.smim.2003.10.003

[33] DelaRosa, O. and Lombardo, E. (2010) Modulation of Adult Mesenchymal Stem Cells Activity by Toll-Like Receptors: Implications on Therapeutic Potential. Mediators of Inflammation, 2010, Article ID: 865601.

[34] Mizgerd, J.P. (2005) Bacterial Lipopolysaccharide Induces Macrophages to Migrate from Inflamed Tissues to Lymph Nodes Using the Adhesion Molecule Mac-1. Volume 106, 9. 